\title{
Nanoparticle Electrocatalysis: Unscrambling Illusory Inhibition and Catalysis
}

\author{
Enno Kätelhön, Lifu Chen, and Richard G. Compton* \\ Department of Chemistry, Physical and Theoretical Chemistry Laboratory, Oxford University, South \\ Parks Road, Oxford, OX1 3QZ, United Kingdom
}

\begin{abstract}
Voltammetry is ubiquitously used in the characterisation of new materials for electrocatalysis. It often sets at least the starting point of any analysis aiming to explore whether electrodes decorated with nano- or other particles feature advanced catalytic behaviour over their bare equivalents. Such analysis bears a substantial and entirely unexpected level of complexity as the mass transport inside the porous layer formed by the particles has a dramatic impact on the measurement. It is shown even for entirely inactive, non-conducting, and non-adsorbing particles, the voltammetry of any electrochemically reversible reaction necessarily varies both between seeming catalysis and apparent irreversible kinetics merely depending on the voltage scan rate. This effect arises from the superposition of thin layer effects and locally enhanced diffusive fluxes inside the porous material with the latter being reminiscent of convergent diffusion and leading to apparent electrochemically-irreversible reactions. While the relevance of thin layer effects in porous materials has been realised before, the seeming irreversible reactions and the understanding of them as result of the internal structure of the modifying layer are, to our knowledge, an entirely new finding. The presented results are counter-intuitive and yet essential for all voltammetric analysis of catalysis at nanostructured interfaces and at porous electrode materials.
\end{abstract}

Keywords: electrocatalysis, nanoparticles, nanoporous electrodes,

\section{Introduction}

Recent years have seen great advances in research on nanostructured electrodes $[1,2$, $3,4,5,6,7]$. Surfaces are often modified with nanoparticles and dramatic changes in the voltammetry may be observed: At individual $[8,9,10]$ or ensembles $[2,8]$ of nanoparticles,

\footnotetext{
*Corresponding author: Richard G. Compton, richard.compton@chem.ox.ac.uk
} 
reaction kinetics, analyte mass transport, and adsorption differ significantly from what is seen at bare electrodes. Observations give rise to high hopes, expectations, and great opportunities setting electrocatalysis at the heart of recent research in material science: Much attention has been attracted by topics including carbon materials [11, 12, 13], titanates and $\mathrm{TiO}_{2}[14]$, nanorods and nanowires $[15,16]$, and functionalised nanomaterials for bio-sensing [17].

Voltammetry is certainly the most commonly used technique in electrochemistry and for decades has been the starting point of almost every electrochemical investigation. This includes studies of electrocatalysis $[18,19,20,21]$ at new surface materials which aim to determine whether a modification alters the speed at which an electrochemical reaction progresses. To this end, at an electrode modified with nanoparticles, one may conduct a simple voltammetric analysis: The electrode is set in contact with a solution comprising the reactant of interest, and, while the electrode current is monitored, the electrode potential is varied linearly with time from a potential at which no reaction occurs to a potential at which the reaction fully driven by the electrode and only limited by the mass transport of the reactant towards it. Intuitively one may then infer that an "earlier" occurrence of a current peak signifies an accelerated reaction and a "later" peak indicates an irreversible reaction. Equally, one may interpret varied peak-to-peak separations in cyclic voltammetry, where the electrode potential is subsequently swept back to its initial value, as changes in the reaction speed: Greater separations suggest slower reaction kinetics as both the forward- and the backward peak occur "later" while smaller separations may be interpreted to result from a more reversible reaction. This work however proves that such experiments feature a considerably greater complexity and a far more rigorous analysis is needed. We exemplarily investigate a reversible electrochemical reaction at a macroelectrode modified with a single layer or layers of inactive spherical nanoparticles which simply serve to 'block' [22] the electrode. Our results show that depending on the voltage scan rate both seemingly accelerated and seemingly retarded reactions are observed! Whilst the former has been previously noted [23, 24, 25, 26] for other porous 
materials, the latter is a new ${ }^{2}$ voltammetric insight and results from locally enhanced diffusive fluxes. The novelty of this work lies hence in the description of the seming irreversible reaction and understanding it as a consequence of the internal structure of the modifying layer. This finding is generic and applies to all electrode modifications with both randomly-oriented nanoparticles and many modifications through self-aligned nanostructures if dense or multiple layers of the material are applied.

\section{Theoretical Model}

Our computational analysis investigates an electrochemically reversible reaction, $A+$ $e \rightleftarrows B$, at a macroelectrode modified with varying numbers of equally sized inactive, i.e. non-conductive ${ }^{3}$, non-adsorbing, and non-electroactive, spheres packed in complete layers (Figure 1a), similar to models previously described in literature [31, 32] on diffusion in porous materials. The solution initially comprises only one of the two species and mass transport solely occurs by virtue of diffusion as the experiment is assumed to be conducted in presence of a sufficient amount of electrolyte screening all electric fields in solution. For modelling purposes, this geometry is simplified along the lines of a diffusion domain approximation [33, 34, 35] (Figure 1b), and evaluated in cylindrical coordinates (Figure 1c). We note that these approximations may impact results, the conclusions drawn yet remain qualitatively unchanged. In fact and as it will be shown below, every electrode modification that approximates a series of volumes will exhibit a qualitatively similar voltammetric response if volumes are weakly-coupled in terms of diffusion and connect the electrode on one side and with the bulk solution on the other. For details of the theoretical model, its implementation and validation [36], and the dimensionless coordinates used, please refer to the Supporting Information. For simplicity and transferability of our results, all data is expressed in dimensionless coordinates as defined in Table 1.

\footnotetext{
${ }^{2}$ The presented results must not be confused with a prior study [27] by this group in which an entirely different structure, namely a conducting porous film, was investigated. prior works of this group. Ban et al. [27] investigated an entirely different structure, namely a conducting porous film which features a very different voltammetric behaviour. In another study, Hepburn et al. [28] model an electrode modified with inactive particles and, similar to the here-presented work, investigate the effect of the modification on the voltammetry. The theoretical model is however distinctly different: While we report effects that result from the complex mass transport within the heterogeneous particle film, Hepburn et al. considered a uniform film. Observed changes in the voltammetric response of the latter electrode are hence very different and, in Hepburn's case, arise from the different diffusion coefficient and an assumed different solubility of the analyte inside the modifying film.

${ }^{3}$ The voltammetry of electrodes modified with conducting porous layers [29, 30, 23, 24] differs significantly from the here considered electrode and requires modelling via a different theoretical model.
} 

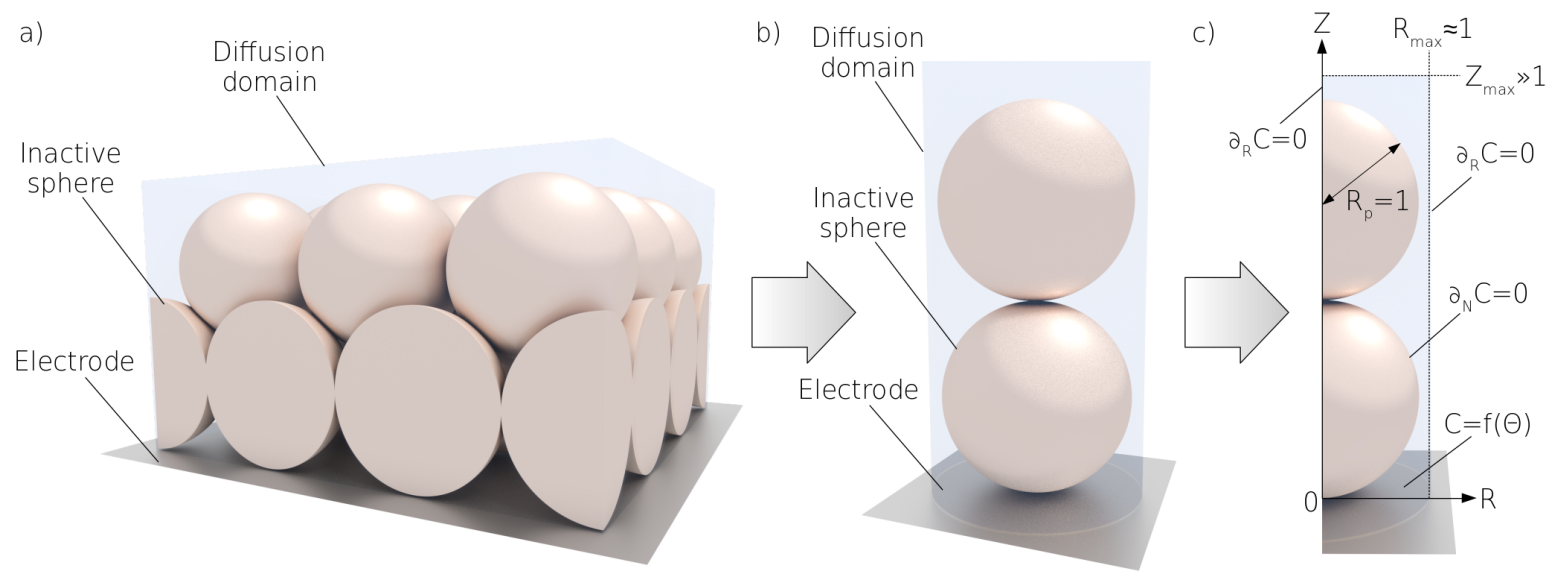

Figure 1: Illustration of the geometry considered. a) The electrode surface is decorated with inactive nanoparticles arranged in a hexagonal packing pattern. b) The structure depicted in a) is approximated as a stack of spheres located centrally in a cylindrical diffusion domain. c) The rotational symmetry of the diffusion domain is exploited to transform the three-dimensional structure into a two-dimensional geometry over which diffusion equation is solved subject to the boundary conditions shown. $R$ and $Z$ signify dimensionless cylindrical coordinates, $C$ is the dimensionless concentration of the reactant, $R_{p}$ is the particle radius, $\theta$ is the dimensionless electrode potential, and $f(\theta)$ represents a Nerstian Dirichlettype boundary condition. The radius of the simulated space, $R_{\max }$, is set to 1.05 in all calculations while $Z_{\text {max }}$ is chosen sufficiently large value to not perturb the simulation results.

\begin{tabular}{c|c|c} 
Parameter & Symbol (dimensioned) & Symbol (dimensionless) and transformation \\
\hline Radial coordinate & $r$ & $R=\frac{r}{r_{p}}$ \\
Axial coordinate & $z$ & $Z=\frac{z}{r_{p}}$ \\
Time & $t$ & $T=\frac{D}{r_{p}^{2}} t$ \\
Potential & $E$ & $\theta=\frac{F}{R T}\left(E-E_{f}^{0}\right)$ \\
Scan rate & $v$ & $\sigma=\frac{r_{p}^{2} F}{D R T} v$ \\
Concentration & $c$ & $C=\frac{c}{c^{*}}$ \\
Flux & $j$ & $J=\int_{S} d A\left(-\frac{\partial C}{\partial Z}\right)$
\end{tabular}

Table 1: Definition of the dimensionless coordinates used throughout the study. $r_{p}$ is the particle radius, $D$ is the diffusion coefficient of both species, $E_{f}^{0}$ is formal potential of the reaction, $c^{*}$ is the bulk concentration of the initially present species, $S$ is the dimensionless surface of the electrode, and $d A$ is a dimensionless surface element.

\section{Results and Discussion}

Figure 2 presents cyclic voltammograms simulated for different electrode modifications and illustrates that, as one might anticipate, the voltammetry changes with the modification while voltammetric features of significance to the characterisation of catalytic materials - and many other electroanalytical applications - vary. The following provides an analysis of how these features, namely peak positions, peak-to-peak separations, and peak heights, are affected by the electrode modification. 


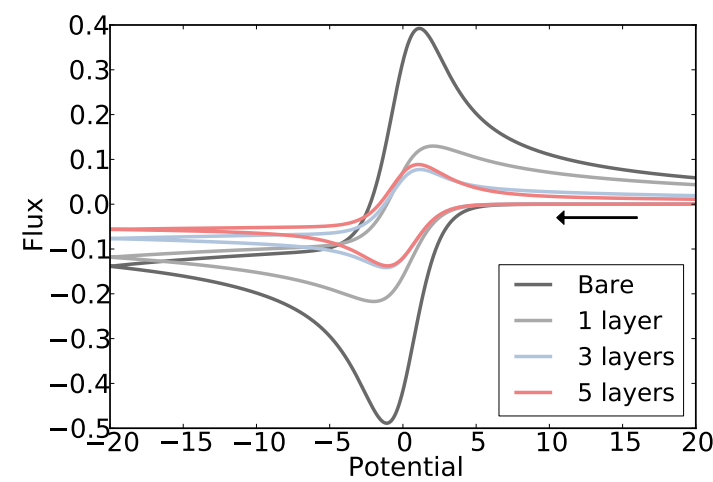

Figure 2: Voltammograms of different electrode geometries calculated for a dimensionless scan rate of 0.1 . The theoretical model is evaluated for a bare macro electrode and compared with models of electrodes decorated with one, three, and five layers of inactive spheres.

Our study commences with an investigation of the peak positions and the resulting peak-to-peak separations, i.e. the absolute difference between the potentials at which the peaks are observed. To this end, we evaluate voltammograms for different electrode modifications as a function of the scan rate as shown in Figure 3. The figure reveals remarkable changes in the positions of the peaks: While at low and high scan rates peak positions approach the positions observed at bare electrodes, there are substantial variations in the intermediate region. At a scan rate of about five, the peak positions for all modified electrodes shift towards the thermodynamic formal potential which in the dimensionless coordinates employed equals to zero, and the peak-to-peak separation falls to only less than half of the value observed at a bare electrode at the same scan rate. In stark contrast and possibly counter-intuitively, at a dimensionless scan rate of 0.1 , a single layer of spheres results in peaks shifted in the opposite direction away from the formal potential while the peak-to-peak separation approximately doubles. Electrodes modified with more than one layer exhibit a similar voltammetry with even more pronounced shifts appearing at lower scan rates. The implications of this result for experimental work are tremendous: If the subtleties of an electrochemical system are not sufficiently recognised, any cyclovoltammetric analysis of an electrode modification may hence, and almost necessarily will, falsely suggest either accelerated or decelerated reaction kinetics independent of the actual kinetics. The factors in Figure 2 and 3 will be present in any particle modified electrode in the absence of any 'active' effects of the spheres. To further emphasise the significance of this finding, we replot the peak-to-peak separations in dimensional coordinates for common experimental set-ups in Figure 4 where a dramatic impact can be seen. A discussion of the peak fluxes that correspond to the same peaks analysed in Figure 3 is provided at the end of the manuscript in Figure 6. 

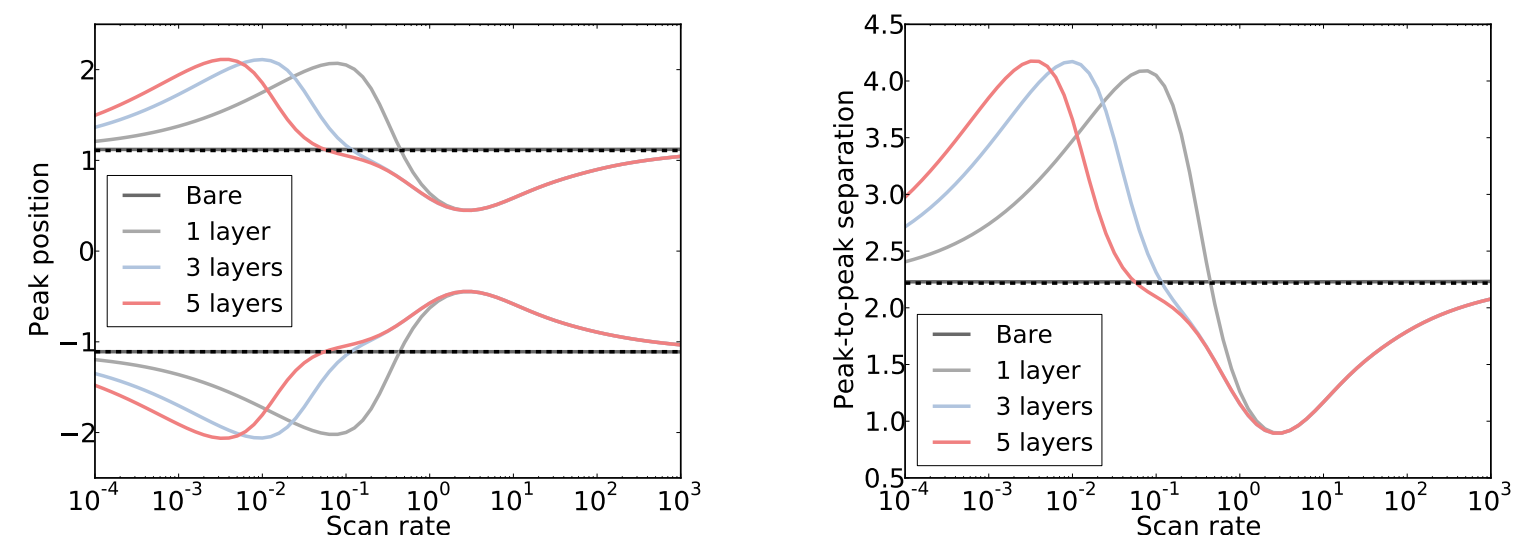

Figure 3: Peak positions and peak-to-peak separations as a function of the scan rate. Dashed black lines indicate the theoretical results for a bare electrode. The voltammetric scan covers a potential range of \pm 20 .

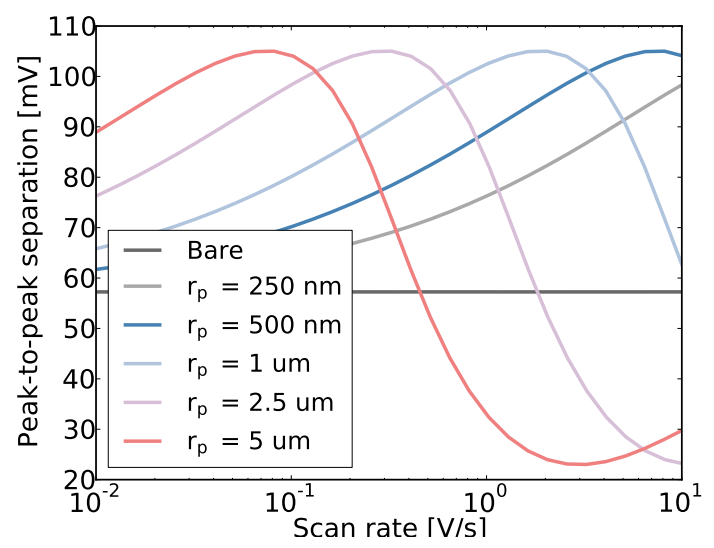

Figure 4: Replotted peak-to-peak separations in dimensional coordinates. Results are shown for monolayers of identical spheres featuring sizes sizes between $250 \mathrm{~nm}$ and $5 \mu \mathrm{m}$, an analyte diffusion coefficient of $10^{-9} \mathrm{~m}^{2} \mathrm{~s}^{-1}$ and a temperature of 298 Kelvin. [figure has been

The factors in Figure 2 and 3 will be present in any particle modified electrode absented by any 'active' effects of the spheres.

To identify the underlying physical principles that lead to the remarkable dependency of the peak position on the scan rate, we analyse the concentration profiles of species $A$ throughout the modelled time interval. Figure 5 depicts the concentration as a function of time and evaluated along a line parallel to the $Z$ - axis and perpendicular to the electrode surface. This line cuts through one layer of spheres deposited on the electrode as illustrated in Figure 5a.

We first focus on the concentration profiles in Figure 5b which correspond to a dimensionless scan rate of 5 at which we have priorly observed a peak shifts towards the formal potential. The concentration profile illustrates that mass transport in the two volumes 
formed by the sphere, the volume confined by the electrode and the sphere (I) and the volume above the sphere (II), are almost entirely decoupled in terms of diffusion. Concentration changes in (I) near the electrode have only little impact on the concentration in (II) above the sphere. In addition, we find that there is hardly any concentration gradient in (I) though the value of the absolute concentration changes with time. The solution in (I) hence remains close to the thermal equilibrium at any time, the concentration not only at the surface but throughout the entire volume approaches Nernst equation, and the voltammetry begins to approach a classical thin layer behaviour [33, 37] with peak positions and a peak-to-peak separation of zero rather than a one-dimensional diffusional voltammogram featuring a peak-to-peak separation of 2.218 , which amounts to approximately $57 \mathrm{mV}$ at room temperature.

Second and much on the contrary, in Figure 5c, we find a completely different mass transport at the slower scan rate of 0.1. On this time scale, the volumes (I) and (II) are weakly coupled in terms of diffusion and the concentration near the sphere in (II) follows the concentration in (I). We can though note a slight delay in response as a result of the much narrowed space available to diffusion where the distance between the sphere and the outer boundary in $R$ is the smallest. The observed voltammetry is hence a superposition of the thin-layer voltammetric signal seen in the previous case and the 'delayed' voltammetric signal that arises from material initially located in (II). Due to the limited diffusive coupling between (I) and (II), the latter material in (II) requires more time to reach the electrode surface and therefore only reacts when the electrode potential has already progressed to a greater absolute over- or underpotential. Consequentially, the forward- and backward peaks occur at potential shifts further away from the formal potential. In between the scan rates of 0.1 and 5 there is a point where both effects, the thin-layer effect and the delayed mass transport from (II), balance out and no potential shift is observed compared to the bare electrode.

Third, the peak positions at high and low scan rates, where the voltammetry approaches the behaviour of a bare electrode, can be readily understood with 'classic' voltammetric theory: At fast scan rates the diffusion field does not spread notably into the porous material formed by the spheres and the mass transport does hence not differ qualitatively from the mass transport to a bare electrode. At slow scan rates, the space near the electrode reaches the thermal equilibrium, which, due to the long time scale, embraces the entire surface modification and equally resembles the diffusion field of a bare electrode.

We next investigate the peak heights of the first peaks as a function of the electrode modification. Figure 7 depicts a different analysis of the same simulated data already 

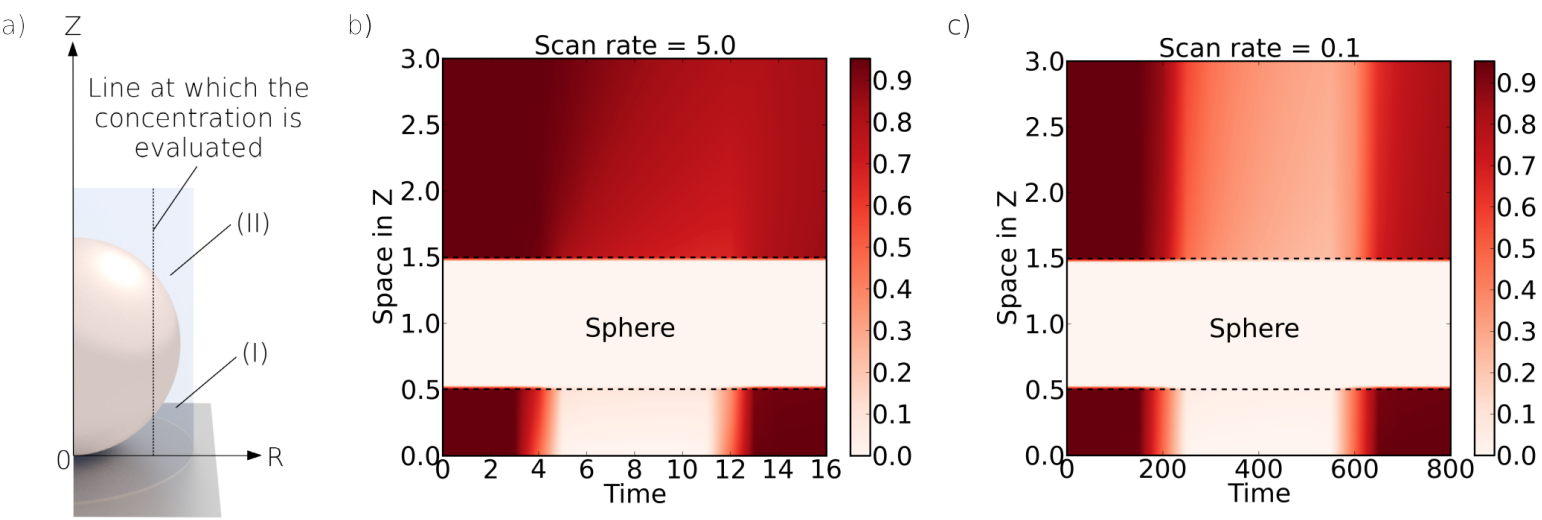

Figure 5: [This figure was amended.]Concentration profiles as a function of $Z$ and time evaluated for two different dimensionless scan rates and one layer of spheres. a) Schematic depiction of the line at which the concentration profile is calculated. b), c) Concentration profiles calculated for scan rates of 5 and 0.1 , respectively. Please note that for the clarity of presentation voltammograms are modelled within a dimensionless potential range of \pm 10 rather than \pm 20 in all other simulations.

been analysed with respect to the peak positions in Figure 3 and 4. as a function of the sean rateThe figure reveals where find reduced peak heights for all modifications and at all scan rates. The greater is the amount of inactive material deposited on the electrode, the more pronounced is the reduction in peak current and and the more it spreads over a greater range of scan rates. This result is much in line with possible expectations as the modification with the inactive material retards the mass transport to the electrode. In addition, for small and large scan rates, peak heights again approach the ones modelled for a bare electrode for the reasons outlined in the previous paragraph: At long times scales, the space near the electrode reaches the steady state which is a thermal equilibrium similar to that at a bare electrode while, at short time scales, the diffusion field does not reach out far enough into the solution to be effected by the electrode modification.

Figure 7 summarises all of the above key findings and contrasts the voltammetry of a bare- and a modified electrode.

\section{Conclusions}

We have proved that detailed modelling is mandatory for any conclusions to be drawn from cyclic voltammetric data of electrodes modified with particles. The experimental system bears a remarkable and quite unexpected level of complexity which arises from the altered mass transport inside the porous layer formed by the deposited particles: Classical thin-layer behaviour or a retarded mass transport within the porous layer may dominate the voltammetry at certain scan rates. If these subtleties were to be ignored, 


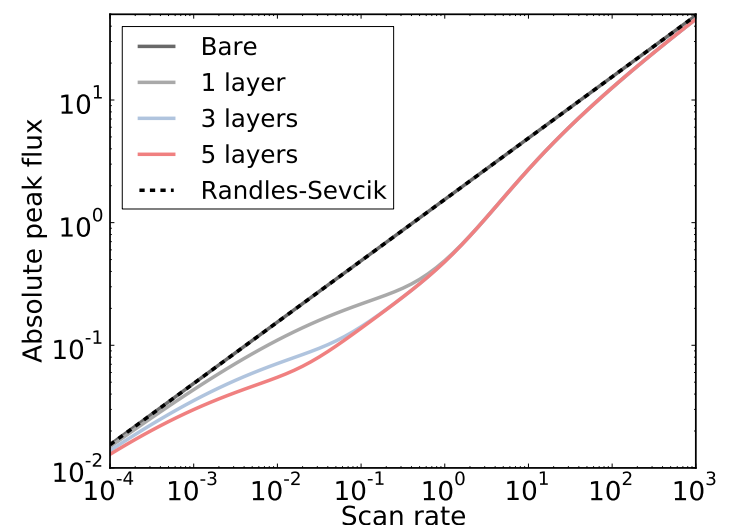

Figure 6: Oxidative peak heights as a function of the scan rate. The case of a bare macro electrode is additionally compared with Randles-Ševčík equation illustrating that both graphs overlap. The voltammetric scan again covers a dimensionless potential range of \pm 20 .

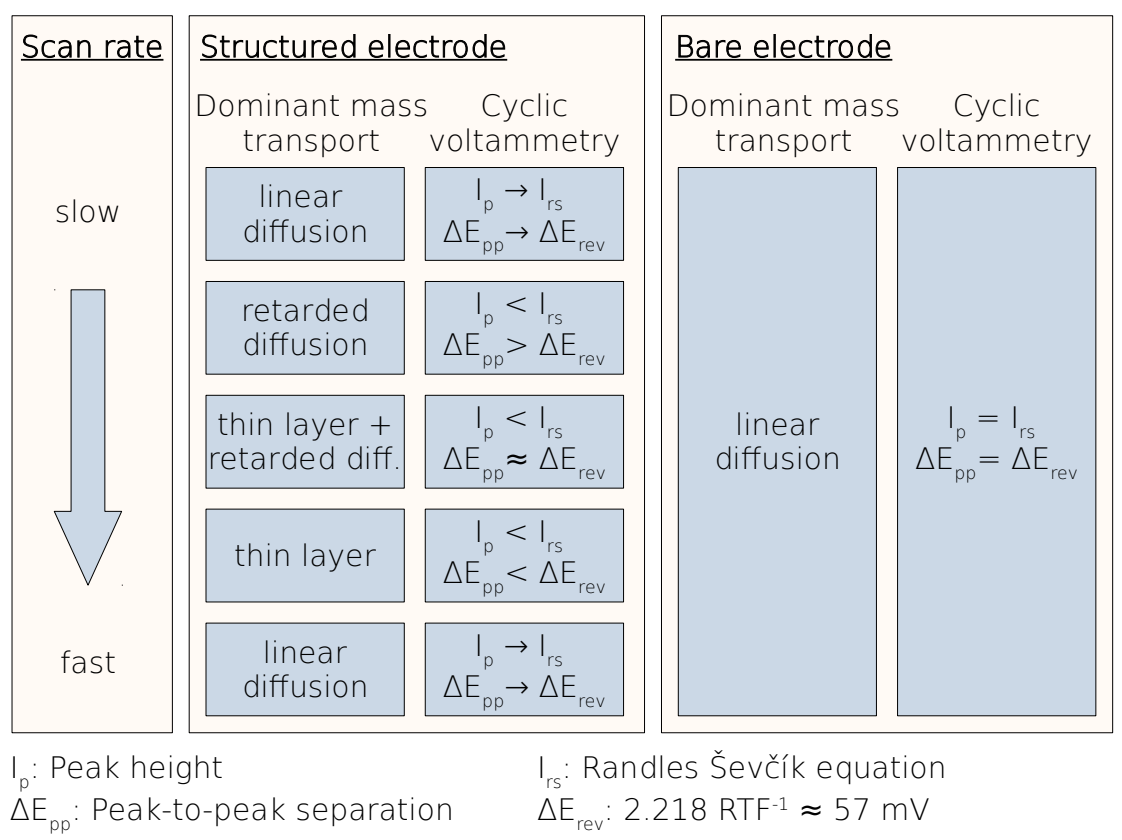

Figure 7: Schematic summary of this work's key findings. While the voltammetry of at bare electrodes follows 'classical' theory in terms of peak height and peak-to-peak separation, modified electrodes exhibit an entirely different voltammetric behaviour. Only at low and a fast scan rates the voltammetry of a bare electrode is approached. Everywhere else, we see an interplay between thin layer voltammetry of analyte in the immediate proximity of the electrode and material that was initially located further away from the electrode and reaches it by retarded mass transport through the porous membrane.

false impressions of electrocatalysis - positive or negative - would be measured and, under many circumstances, a catalytic activity of the modifying nanoparticles would falsely be 
inferred. ${ }^{4}$ While the paramount importance of thin layer effects in porous materials has been realised in prior literature, the seeming irreversible reactions as a result of the internal structure of a modifying layer have, to our knowledge, never before been reported. While Though our current work focuses on reversible voltammetry, we note that similar considerations must be taken into account in any analysis of quasi- and irreversible systems which we intend to address in future publications.

\section{Acknowledgement}

The research leading to these results has received partial funding from the European Research Council under the European Union's Seventh Framework Programme (FP/20072013) / ERC Grant Agreement no. [320403].

\section{Data Availability}

The raw/processed data required to reproduce these findings cannot be shared at this time due to technical or time limitations.

\section{References}

[1] R. G. Compton, G. G. Wildgoose, N. V. Rees, I. Streeter, R. Baron, Design, fabrication, characterisation and application of nanoelectrode arrays, Chemical Physics Letters 459 (1) (2008) $1-17$.

[2] R. W. Murray, Nanoelectrochemistry: Metal nanoparticles, nanoelectrodes, and nanopores, Chemical Reviews 108 (7) (2008) 2688-2720.

[3] Q. Lu, J. G. Chen, J. Q. Xiao, Nanostructured electrodes for high-performance pseudocapacitors, Angewandte Chemie International Edition 52 (7) (2013) 1882-1889.

[4] C. Batchelor-McAuley, E. Kätelhön, E. O. Barnes, R. G. Compton, E. Laborda, A. Molina, Recent advances in voltammetry, ChemistryOpen 4 (3) (2015) 224-260.

\footnotetext{
${ }^{4}$ Rotating disc electrodes are occasionally suggested as a means of overcoming these problems, see for example Langmuir 2012, 28, 5275. The limitations of this approach are well recognised as detailed by D. C. Johnson at al., Electroanalysis, 2002, 15, 165; S.-Z. Qiao et al., ACS Catalysis, 2016, 6, 4720; W. Schuhmann et al., Nano Research, 2014, 7, 71 and overviewed in S. V. Sokolov et al., Applied Materials Today, 2017, 7, 82 .
} 
[5] F. Wang, X. Wu, C. Li, Y. Zhu, L. Fu, Y. Wu, X. Liu, Nanostructured positive electrode materials for post-lithium ion batteries, Energy Environmental Science 9 (2016) 3570-3611.

[6] K. J. Aoki, Voltammetry at a single nano-electrode by varying electrode diameters: Review, Journal of Electroanalytical Chemistry 779 (2016) 7 - 17.

[7] L. Zhang, Z.-J. Zhao, J. Gong, Nanostructured materials for heterogeneous electrocatalytic $\mathrm{CO} 2$ reduction and their related reaction mechanisms, Angewandte Chemie International Edition 56 (38) (2017) 11326-11353.

[8] S. E. F. Kleijn, S. C. S. Lai, M. T. M. Koper, P. R. Unwin, Electrochemistry of nanoparticles, Angewandte Chemie International Edition 53 (14) 3558-3586.

[9] M. V. Mirkin, T. Sun, Y. Yu, M. Zhou, Electrochemistry at one nanoparticle, Accounts of Chemical Research 49 (10) (2016) 2328-2335.

[10] S. V. Sokolov, S. Eloul, E. Kätelhön, C. Batchelor-McAuley, R. G. Compton, Electrode-particle impacts: A users guide, Physical Chemistry Chemical Physics 19 (2017) 28-43.

[11] C. E. Banks, T. J. Davies, G. G. Wildgoose, R. G. Compton, Electrocatalysis at graphite and carbon nanotube modified electrodes: Edge-plane sites and tube ends are the reactive sites, Chemical Communications (2005) 829-841.

[12] L. Wang, M. Pumera, Electrochemical catalysis at low dimensional carbons: Graphene, carbon nanotubes and beyond - a review, Applied Materials Today 5 (2016) $134-141$.

[13] V. C. Hoang, M. Hassan, V. G. Gomes, Coal derived carbon nanomaterials - recent advances in synthesis and applications, Applied Materials Today 12 (2018) 342 - 358.

[14] D. Bavykin, J. Friedrich, F. Walsh, Protonated titanates and $\mathrm{TiO}_{2}$ nanostructured materials: Synthesis, properties, and applications, Advanced Materials 18 (21) (2006) $2807-2824$.

[15] Y.-P. Zhu, Y.-P. Liu, T.-Z. Ren, Z.-Y. Yuan, Self-supported cobalt phosphide mesoporous nanorod arrays: A flexible and bifunctional electrode for highly active electrocatalytic water reduction and oxidation, Advanced Functional Materials 25 (47) (2015) 7337-7347. 
[16] A. Serrà, E. Vallés, Advanced electrochemical synthesis of multicomponent metallic nanorods and nanowires: Fundamentals and applications, Applied Materials Today 12 (2018) $207-234$.

[17] H. Ju, Functional nanomaterials and nanoprobes for amplified biosensing, Applied Materials Today 10 (2018) $51-71$.

[18] R. L. McCreery, Advanced carbon electrode materials for molecular electrochemistry, Chemical Reviews 108 (7) (2008) 2646-2687.

[19] D. Chen, L. Tang, J. Li, Graphene-based materials in electrochemistry, Chemical Society Review 39 (2010) 3157-3180.

[20] E. S. Rountree, B. D. McCarthy, T. T. Eisenhart, J. L. Dempsey, Evaluation of homogeneous electrocatalysts by cyclic voltammetry, Inorganic Chemistry 53 (19) (2014) 9983-10002.

[21] N.-T. Suen, S.-F. Hung, Q. Quan, N. Zhang, Y.-J. Xu, H. M. Chen, Electrocatalysis for the oxygen evolution reaction: recent development and future perspectives, Chemical Society Review 46 (2017) 337-365.

[22] B. A. Brookes, T. J. Davies, A. C. Fisher, R. G. Evans, S. J. Wilkins, K. Yunus, J. D. Wadhawan, R. G. Compton, Computational and experimental study of the cyclic voltammetry response of partially blocked electrodes. Part 1. Nonoverlapping, uniformly distributed blocking systems, The Journal of Physical Chemistry B 107 (7) (2003) 1616-1627.

[23] M. C. Henstridge, E. J. F. Dickinson, M. Aslanoglu, C. Batchelor-McAuley, R. G. Compton, Voltammetric selectivity conferred by the modification of electrodes using conductive porous layers or films: The oxidation of dopamine on glassy carbon electrodes modified with multiwalled carbon nanotubes, Sensors and Actuators B: Chemical 145 (1) (2010) 417 - 427.

[24] M. C. Henstridge, E. J. F. Dickinson, R. G. Compton, Mass transport to and within porous electrodes. Linear sweep voltammetry and the effects of pore size: The prediction of double peaks for a single electrode process, Russian Journal of Electrochemistry 48 (6) (2012) 629-635.

[25] E. O. Barnes, X. Chen, P. Li, R. G. Compton, Voltammetry at porous electrodes: A theoretical study, Journal of Electroanalytical Chemistry 720-721 (2014) 92 - 100. 
[26] H. T. Chan, E. Kätelhön, R. G. Compton, Voltammetry using multiple cycles: Porous electrodes, Journal of Electroanalytical Chemistry 799 (2017) 126 - 133.

[27] Z. Ban, E. Kätelhön, R. G. Compton, Voltammetry of porous layers: Staircase vs analog voltammetry, Journal of Electroanalytical Chemistry 776 (2016) 25 - 33.

[28] Hepburn, William G. and Batchelor-McAuley, Christopher and Tschulik, Kristina and Barnes, Edward O. and Torabi Kachoosangi, Roohollah and Compton, Richard G., Diffusional transport to and through thin-layer nanoparticle film modified electrodes: Capped CdSe nanoparticle modified electrodes, Physical Chemistry Chemical Physics 18 (2014) 18034-18041.

[29] I. Streeter, G. G. Wildgoose, L. Shao, R. G. Compton, Cyclic voltammetry on electrode surfaces covered with porous layers: An analysis of electron transfer kinetics at single-walled carbon nanotube modified electrodes, Sensors and Actuators B: Chemical 133 (2) (2008) $462-466$.

[30] M. J. Sims, N. V. Rees, E. J. F. Dickinson, R. G. Compton, Effects of thin-layer diffusion in the electrochemical detection of nicotine on basal plane pyrolytic graphite (bppg) electrodes modified with layers of multi-walled carbon nanotubes (mwcntbppg), Sensors and Actuators B: Chemical 144 (1) (2010) 153 - 158.

[31] M. R. Newton, K. A. Morey, Y. Zhang, R. J. Snow, M. Diwekar, J. Shi, H. S. White, Anisotropic diffusion in face-centered cubic opals, Nano Letters 4 (5) (2004) 875-880.

[32] V. M. Volgin, A. D. Davydov, T. B. Kabanova, Calculation of effective diffusion coefficient in a colloidal crystal by the finite-element method, Russian Journal of Electrochemistry 48 (8) (2012) 817-834.

[33] R. G. Compton, C. E. Banks, Understanding voltammetry, second edition, Imperial College Press, 2011.

[34] H. Reller, F. Kirowa-Eisner, E. Gileadi, Ensembles of microelectrodes: A digitalsimulation, Journal of Electroanalytical Chemistry and Interfacial Electrochemistry 138 (1) (1982) $65-77$.

[35] C. Amatore, J. Savéant, D. Tessier, Charge transfer at partially blocked surfaces: A model for the case of microscopic active and inactive sites, Journal of Electroanalytical Chemistry and Interfacial Electrochemistry 147 (1) (1983) 39 - 51. 
[36] E. Kätelhön, R. G. Compton, Testing and validating electroanalytical simulations, Analyst 140 (2015) 2592-2598.

[37] A. J. Bard, L. R. Faulkner, Electrochemical methods: Fundamentals and applications, John Wiley \& Sons, Inc, 2001. 